\title{
Optical properties of $4 \AA$ single-walled carbon nanotubes inside the zeolite channels studied from first principles calculations
}

\author{
X.P. Yang, H.M. Weng, and Jinming Dong \\ Department of Physics and National Laboratory of Solid State Microstructures, \\ Nanjing University, Nanjing 210093, P. R. China
}

(Dated: 16 July 2007)

\begin{abstract}
The structural, electronic, and optical properties of $4 \AA$ single-walled carbon nanotubes (SWNTs) contained inside the zeolite channels have been studied based upon the density-functional theory in the local-density approximation (LDA). Our calculated results indicate that the relaxed geometrical structures for the smallest SWNTs in the zeolite channels are much different from those of the ideal isolated SWNTs, producing a great effect on their physical properties. It is found that all three kinds of $4 \AA$ SWNTs can possibly exist inside the Zeolite channels. Especially, as an example, we have also studied the coupling effect between the $\mathrm{ALPO}_{4}-5$ zeolite and the tube $(5,0)$ inside it, and found that the zeolite has real effects on the electronic structure and optical properties of the inside $(5,0)$ tube.
\end{abstract}

PACS numbers: 78.67.Ch, 73.22. -f, 78.40.Ri 


\section{INTRODUCTION}

In the past decade carbon nanotubes (CNTs) [1-5] had been extensively investigated, which is motivated both by their electrical and mechanical properties as well as by their potential applications in future's nanostructured materials. For example, nanotubes are ideal model systems for studying the transport of electrons in one dimension. Their unique electronic and mechanical properties are proved to be a rich source of new fundamental physics and also make CNTs promising candidates as nanoscale wires, transistors and sensors.

The single-walled carbon nanotube is the simplest type of carbon nanotubes, discovered first by Iijima group [6] in 1993, which is composed of rolled up 2D-graphite sheet. The carbon atoms on the SWNT are arranged in a helical line around its axis. It is well-known that diameter of the SWNTs strongly affects their physical properties, and it has been proved [7] that the calculated curvature energies are inversely proportional to the square of tube radius. The variation of the band gaps of the semiconducting SWNTs with their radius is found to follow the simple rule based on the zone folding theory only for larger diameter tubes, but to differ from it for the tubes with smaller radius. So, it is greatly interested, both theoretically and experimentally [8-11], to study the physical properties of the SWNTs with possible smallest diameters.

The smallest SWNTs with $4 \AA$ diameter have been recently produced in the $1 \mathrm{~nm}$-sized channels of the $\mathrm{ALPO}_{4}-5$ single crystals (AFI in the zeolite terminology) [12], which have the following possible chiral geometrical structures: zigzag $(5,0)$ (diameter, $d=3.93 \AA$ ), armchair $(3,3)(d=4.07 \AA)$ and chiral $(4,2)(d=4.14 \AA)$. For such small nanotubes, their large curvature leads to a hybridization of $\sigma^{*}$ and $\pi^{*}$ orbitals [13], which has great effects on their electronic structure. For example, unlike larger diameter SWNTs, which can be either metallic or semiconducting, depending only on their helicity, now the zigzag $(5,0)$ tube becomes a metal. Therefore, it is an interesting and also a great challenge to investigate experimentally and theoretically physical properties of these smallest SWNTs.

It is known that the AFI is a type of microporous crystal with one-dimensional channels packed in hexagonal arrays. Its framework consists of alternate tetrahedra of $\left(\mathrm{ALO}_{4}\right)^{-}$and $\left(\mathrm{PO}_{4}\right)^{+}$. The AFI single crystals are transparent from the near infrared to the ultraviolet region. Although the AFI is transparent in the specific frequency range, it does not mean, however, that the AFI has no any effect on the electronic and optical properties of the SWNTs inside it. Therefore, it is interesting to see if the AFI crystal has really significant effects on the physical properties of the smallest SWNTs inside it.

In this paper, we use the first-principles calculation to study the structural, electronic and optical properties of the $4 \AA$-diameter SWNTs inside the AFI single crystal. The geometrical structures of all isolated three kinds of the smallest tubes are fully relaxed, and compared with other theoretical results $[14,15]$. Especially, as an example, the effects of the zeolite on the electronic structure and optical absorption of the zigzag $(5,0)$ tube have been studied.

\section{COMPUTATIONAL METHOD AND DETAILS}

The total energy plane-wave pseudopotential method has been used in our calculations within the framework of local density approximation (LDA), in which the exchangecorrelation energy of the Ceperley and Alder form [16] was included. The ion-electron interaction is modeled by ultra-soft local pseudopotentials of the Vanderbilt form [17] for the carbon atoms with maximum plane wave cut-off energy of $280 \mathrm{eV}$. The plane-wave 
pseudopotential program, CASTEP [18], is used on the selected systems.

The tube bundle in the channels of AFI is modeled by using a supercell geometry [13], so that the tubes are aligned in a hexagonal array with the closest distance between the adjacent tubes being $13.726 \AA$, which is the same as that between the adjacent axis of the AFI channels, and found to be a larger enough to prevent the tube-tube interactions. The space group was $P_{1}$ for the computational models involved in this paper. Firstly, we optimized the supercell of the SWNTs employing BFGS geometry optimization scheme $[19,20]$, and the Monkhorst-Pack scheme [21] with a distance of $0.04 / \AA$ between the sampling points in the reciprocal space. The BFGS scheme allows us to specify constraints on the lattice constants and angles before calculation and optimize the cell during the course of calculation. After the final self-consistency cycle, the remaining forces on all atoms were less than $0.03 \mathrm{eV} / \AA$, and the remaining stress was less than $0.05 \mathrm{GPa}$. Secondly, the calculations on band structure and optical properties are carried out on the relaxed tube bundles. The calculation of energy band structure in reciprocal space are performed over $21 k$ points along the tube direction, and in the calculation of the polarized absorption spectra the Monkhorst-Pack scheme with a distance of $0.02 / \AA$ between points is used for the sampling of reciprocal space. In addition, we also carry out the same calculations on three kinds of tube bundles by using the Troullier-Martin norm-conserving nonlocal pseudopotentials [22] in the Kleinman-Bylander form [23] for comparison, and found that obtained results by using the two different kinds of methods are equivalent to each other.

Also, in order to understand the effects of the AFI crystal on tubes inside its channels, we modeled the combined structure of the AFI crystal with zigzag $(5,0)$ tubes in its channels

as shown in Fig. 1. The same lattice constant and symmetry group as those without the AFI crystal are taken for this combined structure, in which the geometrical structures of the zigzag $(5,0)$ tubes had been first relaxed. Here, the reason of selecting zigzag $(5,0)$ tube from the three kinds of SWNTs is because its lattice constant along the tube direction $(\mathrm{c}=$ $4.23 \AA$ ) is about half that of the AFI crystal $(\mathrm{c}=8.484 \AA)$, which makes the AFI structure to match more easily with the zigzag $(5,0)$ tubes in its channels than with other kinds of SWNTs. The total atom numbers in one supercell of the combined structure is 112 (72 for the AFI crystal and 40 for zigzag tube), and so only ultra-soft pseudopotentials can be used in our calculation for this combined structure, taking into account the computational time and cost. The combined structure has been entirely relaxed by the same BFGS geometry optimization scheme.

\section{RESULTS AND DISCUSSIONS}

\section{A. Tube bundles $(5,0),(3,3)$ and $(4,2)$}

The obtained structure parameters for the fully relaxed pure tube bundle are shown in Table 1. The radius of each tube is slightly larger than that from an ideal rolling of a graphite sheet, while the lattice constant along the tube axis is smaller than that of a rolledup graphite. We also calculated the bond lengths of these tubes, and find that the average bond length along the tube axis is shorter than those of the strained bonds in the direction of the circumference. In addition, we find the cohesive energies per carbon atom for three kinds of nanotubes are similar to each other, with only a slight difference of $E_{(4,2)}<E_{(3,3)}<E_{(5,0)}$, while the radius of these tubes are in the reverse order, $r_{(4,2)}>r_{(3,3)}>r_{(5,0)}$. Our results of the geometry optimization are similar to those obtained by others $[14,15]$. The calculated 
electronic band structures for the three SWNTs are shown in Fig. 3, in which the zero of energy is set at the top of the valence band. High-symmetry points in the brillouin zone (BZ) include: $\Gamma=(0,0,0)$ and $\mathrm{X}=(0,0,0.5)$. We also find the zigzag $(5,0)$ tubes are more sensitive to the structure optimization than the armchair $(3,3)$ and the chiral $(4,2)$ tubes in our calculations. It is seen from Fig. 3 that the zigzag $(5,0)$ tube now becomes metallic due to its heavy curvature effects, which is also in consistent with other first-principles calculations $[14,15]$. In addition, the semiconducting $(4,2)$ tube now has a small indirect energy gap of $0.25 \mathrm{eV}$ rather than the direct one of $2 \mathrm{eV}$ obtained from the zone-folding. The armchair $(3,3)$ tube is still metallic, as expected.

Our calculated results for the absorption spectra polarized parallel to the tube direction are given in Fig. 4 and Table 2. A Gaussian smearing of $0.1 \mathrm{eV}$ is used in the calculation of the optical spectra. It is seen from them that there are three strong peaks at $1.48 \mathrm{eV}, 2.88$ $\mathrm{eV}, 3.43 \mathrm{eV}$ and a weaker peak near $2.5 \mathrm{eV}$ for the zigzag $(5,0)$ tube; a sharp peak at 3.3 $\mathrm{eV}$, and a weaker peak at $2.87 \mathrm{eV}$ for the armchair $(3,3)$ tube; and a strong peak at 2.24 $\mathrm{eV}$, and two weaker peaks at $3.04 \mathrm{eV}$ and $3.8 \mathrm{eV}$ for the chiral $(4,2)$ tube. Comparing with the experimental data [24], we can identify the feature A $(1.37 \mathrm{eV})$ is contributed from the peak at $1.48 \mathrm{eV}$ for $(5,0)$ tube, and the feature $\mathrm{B}(2.1 \mathrm{eV})$ from the peak at $2.24 \mathrm{eV}$ for $(4,2)$ tube and also the peak at $2.5 \mathrm{eV}$ for $(5,0)$ tube. Finally, the feature $\mathrm{C}(3.1 \mathrm{eV})$ is coming from all the three kinds of tubes.

For comparison, the same kind of calculation is performed on the three tubes by using the Troullier-Martin norm-conserving non-local pseudopotentials in the Kleinman-Bylander form. It is found that the calculated results are the same as those listed above, showing the suitability of the ultra-soft local pseudopotentials used in our calculations. From the comparison between our calculated results and the experimental observations, it is concluded that all the three kinds of $4 \AA$ tubes can possibly exist in the channels of the AFI crystal. In addition, we find our results for the absorption peaks are roughly the same as those obtained by others except for our peak position are a little blue-shifted compared with those in ref. $[14,15]$. In order to identify correctness of our calculated results, the full potential linearized augmented plane-wave (FP-LAPW) [25] calculation has been performed on the zigzag $(5,0)$ tubes, and obtained result is shown in Fig. 4d and Table 2. A Gaussian smearing of $0.05 \mathrm{eV}$ is used in the FP-LAPW calculation of the optical spectra. Comparing Fig. 4d with 4a, we can find that our results on $(5,0)$ tube obtained by the ultra-soft pseudopotentials is much closer to those by the FP-LAPW method, especially for peak positions.

\section{B. The combined structure of the AFI crystal with the relaxed zigzag $(5,0)$ tube inside the channels}

In order to see the AFI crystal effect, here, we also carry out the first-principles calculations on entirely relaxed combined structure of the AFI crystal with the relaxed zigzag $(5,0)$ tubes inside the channels. Their structure parameters are shown in Table 3 . The calculated energy band structure and optical absorption spectra polarized parallel to the channel direction are shown in Fig. 5 and 6, respectively, in which we used a Gaussian smearing of $0.1 \mathrm{eV}$ for optical spectra.

Comparing Fig. 5 with Fig. 3a, we can find the energy bands close to the Fermi level come still from the $(5,0)$ tube since AFI crystal has a larger energy gap. However, due to the AFI crystal, the both conduction and valence bands now become closer to the Fermi surface. It is also obvious that the energy dispersion along $\Gamma-\mathrm{X}$ direction becomes much 
less than before, meaning the electron wave functions less extended. Especially, the bands at $\mathrm{X}$ point have been much affected in contrast to at the $\Gamma$ point due to the doubling of the period imposed by the zeolite, which causes the tube-like bands close to Fermi level in Fig. 5 to be folded at the $\mathrm{X}$ boundary. In addition, a lot of energy bands coming from AFI exist now in the valence bands lower than $-2.7 \mathrm{eV}$ for the combined structure, and in the energy region of conduction band from 2 to $4 \mathrm{eV}$ some new conduction bands are found, which are energy bands of the pure $(5,0)$ tube moved down from those higher than $4 \mathrm{eV}$. These effects, said above, on the electronic structure of the pure $(5,0)$ tube will be shown in the future's relevant experiments. From Fig. 6, it is seen that there exists a big change for the optical spectra in an energy region from 7.0 to $12.5 \mathrm{eV}$, in which appear several high and sharp peaks. The inset of Fig. 6 shows the allowed dipole transitions in the energy region lower than $4.0 \mathrm{eV}$, in which only a slightly change has been found for the spectra due to effect of the AFI crystal, as expected. It is seen from the inset, the first peak is blue-shifted to the relative higher frequency, and the other peaks are oppositely red-shifted to the lower frequencies. So, the AFI crystal has real effects on the electronic structure and optical absorption spectra of the $(5,0)$ tube bundle in it.

In ref. 14, the effective medium theory is used to calculate the effective dielectric constant of the zeolite-SWNT composite by taking into account the zeolite effects. They thought that the van der Waals interaction between nanotube and zeolite is very weak, as well as the influence of the zeolite on the electronic structure of the nanotube. Therefore, they in fact neglected this interaction, and treated the zeolite-nanotube composite as a homogeneous material with an effective dielectric constant. However, our work really takes into account the interaction between the AFI and $(5,0)$ tubes by the first-principles calculation, and we find that above approximation is justified in the long-wavelength limit, and in general, the AFI has real effect on the absorption spectra.

In our calculation, some factors are not taken into account, for example, the impurities lying between carbon nanotubes and the AFI crystal generated experimentally in the course of the pyrolysis of tripropylamine (TPA) molecules in the AFI channels, which is an interesting problem, and needs more experimental and theoretical investigations in future.

Acknowledgment: This work was supported by the Natural Science Foundation of China under Grant No. 10074026. The authors acknowledge also support from a Grant for State Key Program of China through Grant No. 1998061407.

1 S. Iijima, Nature (London) 354, 56 (1991)

2 T.W. Ebbesen, P.M. Ajayan, Nature (London) 358, 220 (1992)

3 M. Endo, K. Takeuchi, K. Kobori, K. Takahashi, H. W. Kroto, A. Sarkar, Carbon 33, 873 (1995)

4 L.C. Qin, S. Iijima, Mater. Lett. 30, 311 (1997)

5 J. Kong, H.T. Soh, A.M. Cassell, C.F. Quate, H. Dai, Nature (London) 395, 878 (1998)

6 S. Iijima, T. Ichlhashi, Nature 363, 603-5 (1993)

7 O. Gülseren, T. Yildirim, S. Ciraci, Phys. Rev. B 65, 153405 (2002)

8 M.S. Dresselhaus, G. Dresselhaus, P.C. Eklund, Fullerenes and Carbon Nanotubes (Academic, San Diego, 1996)

9 S. Sawada, N. Hamada, Solid State Commun. 83, 917 (1992)

10 P.M. Ajayan, S. Iijima, Nature (London) 358, 23 (1992) 
11 L.F. Sun, S.S. Xie, W. Liu, W.Y. Zhou, Z.Q. Liu, D.S. Tang, G. Wang, L.X. Qian, Nature (London) 403, 384 (2000)

12 N. Wang, Z.K. Tang, G.D. Li, J.S. Chen, Nature (London) 408, 50 (2000).

13 X. Blase, Lorin X. Benedict, Eric L. Shirley, Steven G. Louie, Phys. Rev. Lett. 72, 1878 (1994)

14 M. Machon, S. Reich, C.Thomsen, Phys. Rev. B 66, 155410 (2002)

15 H.J. Liu, C.T. Chan, Phys. Rev. B 66, 115416 (2002)

16 D. M. Ceperley, B. J. Alder, Phys. Rev. Lett. 45, 566-569 (1980)

17 A.M. Rappe, K.M. Rabe, E. Kaxiras, J.D. Joannopoulos, Phys. Rev. B 41, 1227-1230 (1990)

18 CASTEP is an ab initio program with plane-wave pseudopotential package distributed by MSI.

19 T.H. Fischer, J. Almlof, J. Phys. Chem. 96, 9768-9774 (1992)

20 W.H. Press, S.A. Teukolsky, W.T. Vetterling, B.P. Flannery, Numerical Recipies in C, (Cambridge University Press, Cambridge, 1992)

21 H.J. Monkhorst, J.D. Pack, Phys. Rev. B 13, 5188 (1976)

22 N. Troullier, J.L. Martins, Solid State Commun. 74, 13 (1990); Phys. Rev. B 43, 1993 (1991)

23 L. Kleinman, D.M. Bylander, Phys. Rev. Lett. 48, 1425 (1982)

24 Z.M. Li, Z.K. Tang, H.J. Liu, N. Wang, C.T. Chan, R. Saito, S. Okada, G.D. Li, J.S. Chen, N. Nagasawa, S. Tsuda, Phys. Rev. Lett. 87, 127401 (2001)

25 P. Blaha, K. Schwarz, G.K.H. Madsen, D. Kvasnicka, J. Luitz, WIEN2k, An Augmented Plane Wave + Local Orbitals Program for Calculating Crystal Properties (Karlheinz Schwarz, Techn. Universitat Wien, Austria, 2001.ISBN 3-9501031-1-2) 


\section{Table Captions}

Table 1. Parameters of three kinds of SWNTs in a $13.726 \AA \times 13.726 \AA \times \mathrm{C}_{l} \AA$ hexagonal supercell. The parameters (a, b, c, $\alpha, \beta, \gamma)$ are defined as in Fig. 2.

\begin{tabular}{|l|l|l|l|l|l|l|l|l|}
\hline Tube & $\begin{array}{l}\text { diameter } \\
\text { (relaxed) }\end{array}$ & $\begin{array}{l}\text { lattice constant } \\
\mathrm{C}_{l} \\
(\text { relaxed })\end{array}$ & $\mathrm{a}$ & $\mathrm{b}$ & $\mathrm{c}$ & $\alpha$ & $\beta$ & $\gamma$ \\
\hline$(5,0)$ & 4.03 & 4.23 & 1.397 & 1.440 & & 120.0 & 110.9 & \\
\hline$(3,3)$ & 4.17 & 2.445 & 1.420 & 1.429 & & 116.1 & 118.9 & \\
\hline$(4,2)$ & 4.24 & 11.204 & 1.426 & 1.408 & 1.432 & 113.8 & 119.0 & 118.7 \\
\hline
\end{tabular}

Table 2. The calculated parallel polarized absorption spectra of the tube bundles. For comparison, also listed are the experimental results.

\begin{tabular}{|l|l|l|l|l|}
\hline \multirow{2}{*}{$\begin{array}{l}\text { Experiment } \\
\text { Ref. } 24)\end{array}$} & \multicolumn{2}{|l|}{ Ultra-soft pseudopotentials } & \multirow{2}{*}{$\begin{array}{l}\text { FP-LAPW } \\
(5,0)\end{array}$} \\
\cline { 2 - 4 } & $(5,0)$ & $(3,3)$ & $(4,2)$ & \\
\hline (S) 1.2 & & & & \\
\hline (A) 1.37 & 1.48 & & & 1.43 \\
\hline (B) 2.1 & 2.5 & & 2.24 & 2.52 \\
\hline (C) 3.1 & $2.88,3.43$ & $2.87,3.3$ & $3.04,3.8$ & $2.98,3.33$ \\
\hline
\end{tabular}


Table 3. Parameters of the combined structure of AFI crystal with the zigzag $(5,0)$ tube inside the channels. Also listed in it are the calculated parallel polarized absorption peaks of the tube bundles in $0-4 \mathrm{eV}$ region.

\begin{tabular}{|l|l|l|}
\hline$(5,0)$ tube & non-relaxed & relaxed \\
\hline lattice constant $\mathrm{C}_{l}$ & 8.484 & 8.494 \\
\hline diameter & 4.03 & 4.02 \\
\hline $\mathrm{a}, \mathrm{b}, \alpha, \beta$ & $1.397,1.440,120.0,110.9$ & $1.401,1.438,120.2,110.6$ \\
\hline Absorption peaks & & $1.55,2.38,2.77,3.33$ \\
\hline
\end{tabular}




\section{Figure Captions}

FIG. 1. The model of the AFI crystal structure with the zigzag $(5,0)$ tubes inside the channels, viewed along the [001] direction.

FIG. 2. Geometry parameters of the $4 \AA$-diameter nanotubes.

FIG. 3. Calculated energy band structure for three kinds of nanotubes. (a) zigzag $(5,0)$, (b) armchair $(3,3)$, and (c) chiral $(4,2)$. The Fermi level is set at zero.

FIG. 4. Calculated absorption spectra polarized parallel to the tube direction for (a) zigzag $(5,0)$, (b) armchair $(3,3)$, and (c) chiral $(4,2)$ tubes. (d) The FP-LAPW all-electron calculation results for the zigzag $(5,0)$ tube.

FIG. 5. Calculated energy band structure for the entirely relaxed combined structure of the AFI crystal with the relaxed zigzag $(5,0)$ tubes inside the channels. The Fermi level is set at zero.

FIG. 6. Calculated absorption spectra polarized parallel to the channel direction for the relaxed pure zigzag $(5,0)$ (circle), and the entirely relaxed combined structure of the AFI crystal with the

relaxed zigzag $(5,0)$ tubes inside its channels (star). Inset is the absorption spectra in the energy region lower than $4.0 \mathrm{eV}$. 


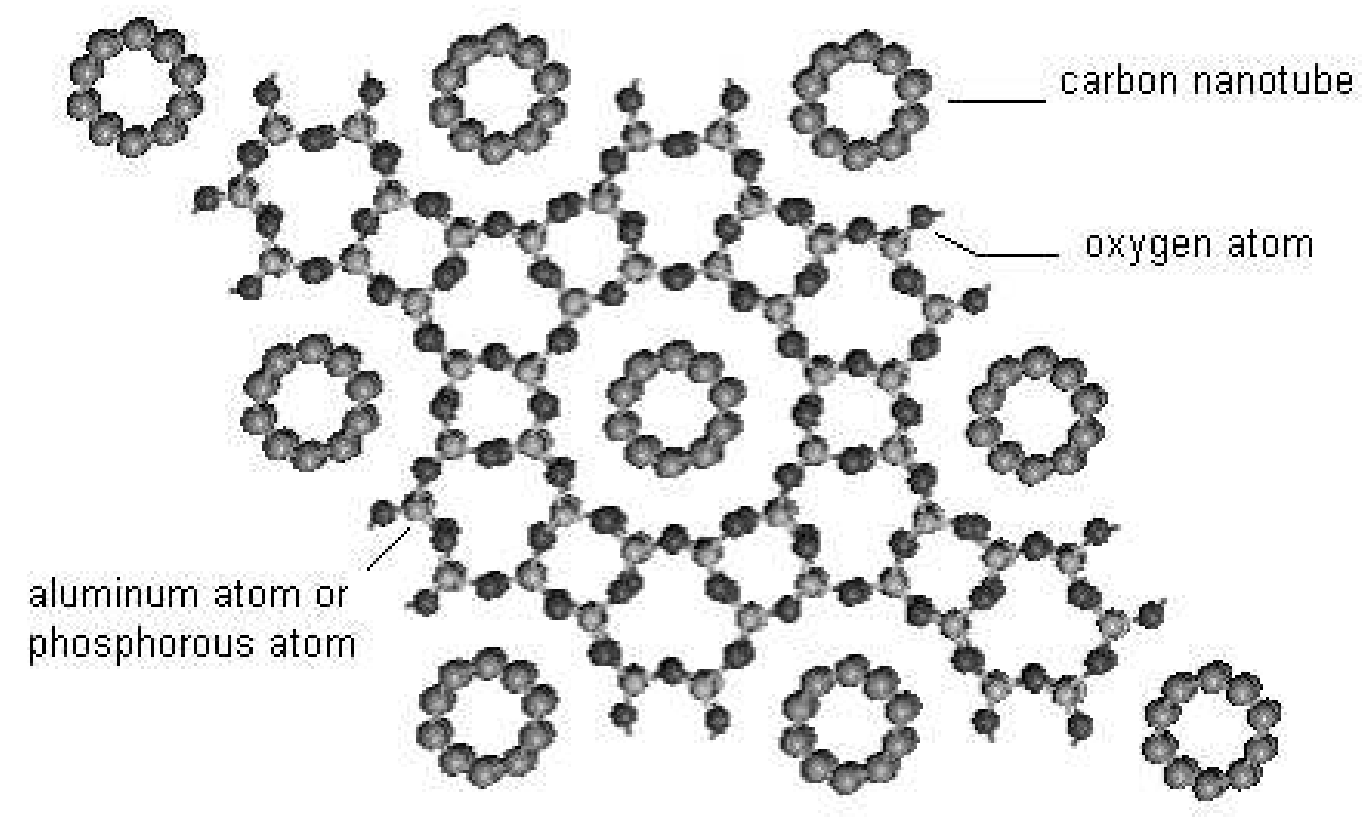




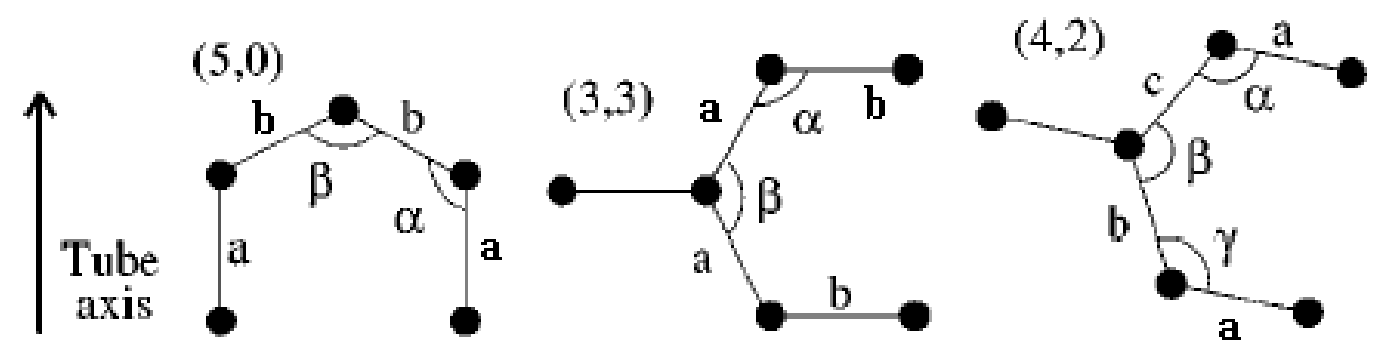




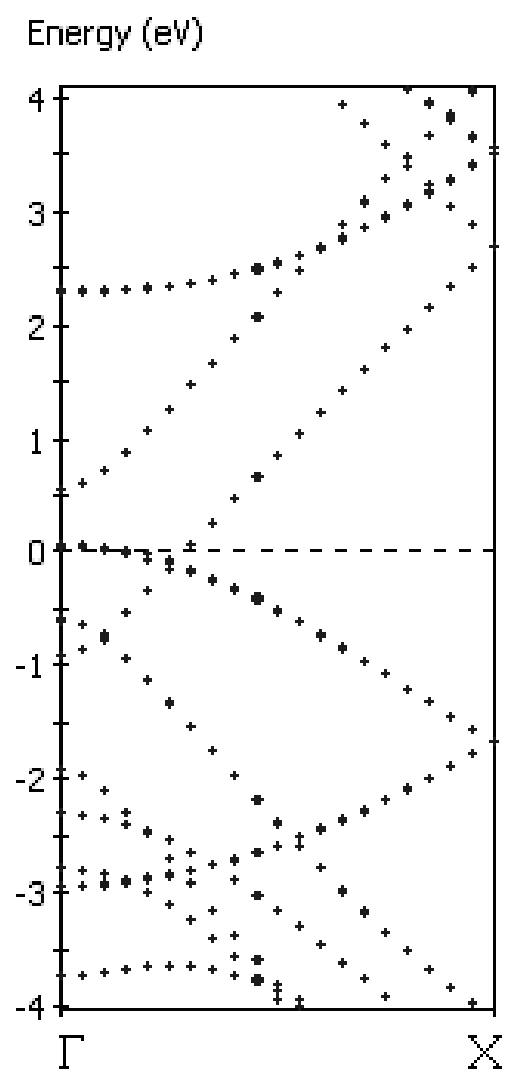

(a) 


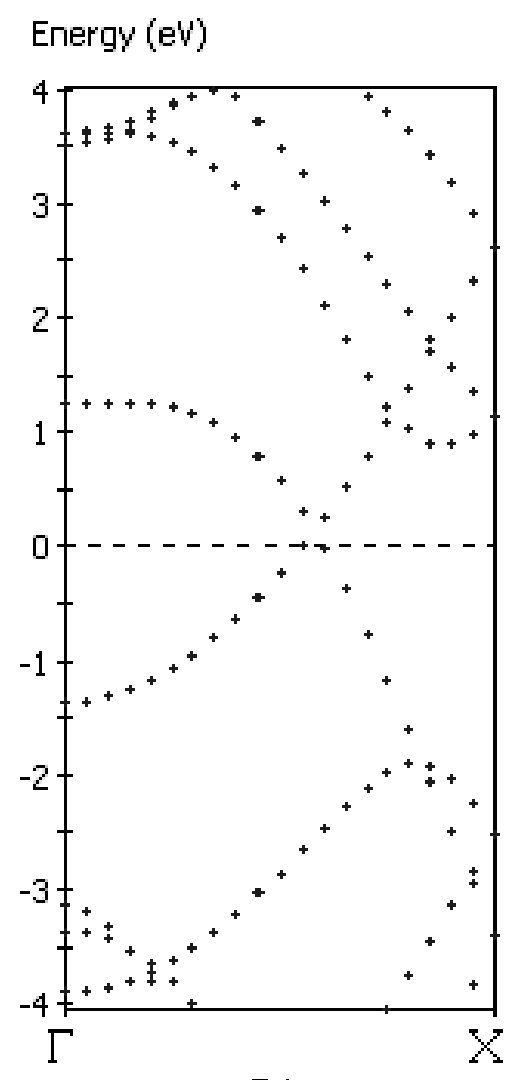

(b) 


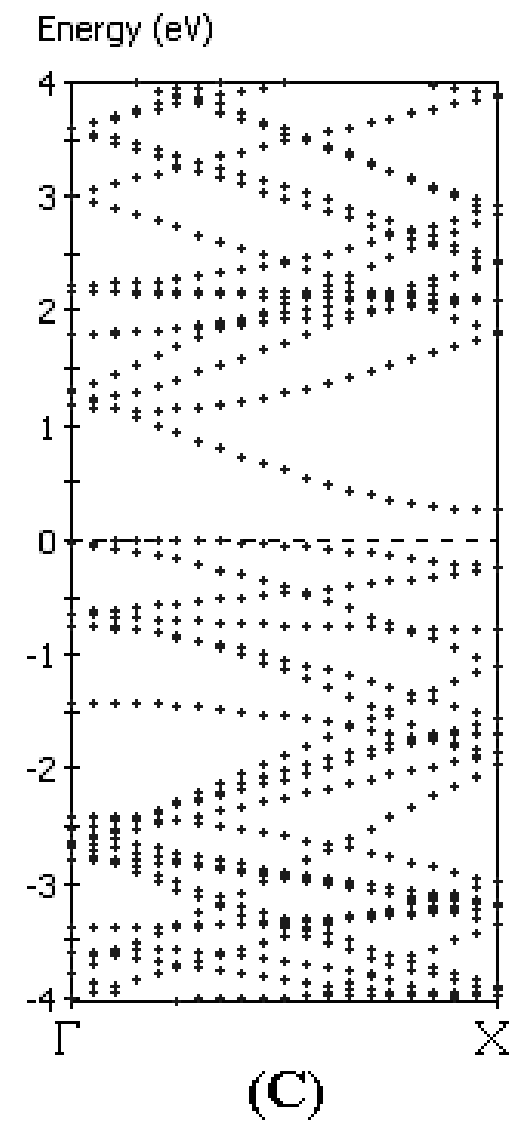




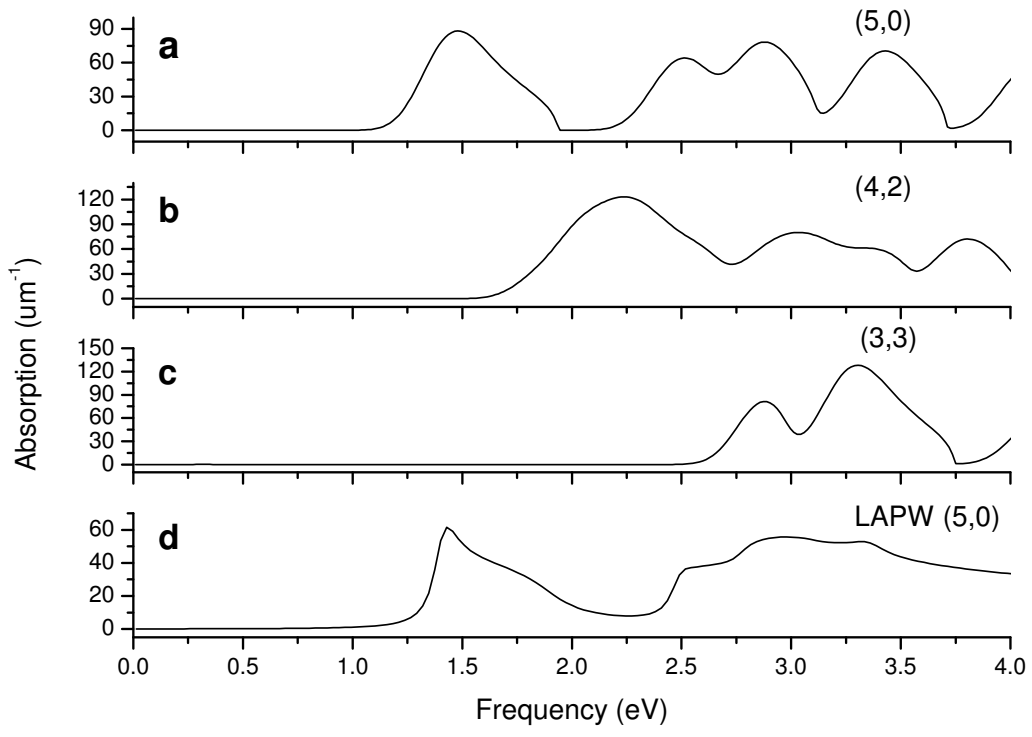


Energy (eV)

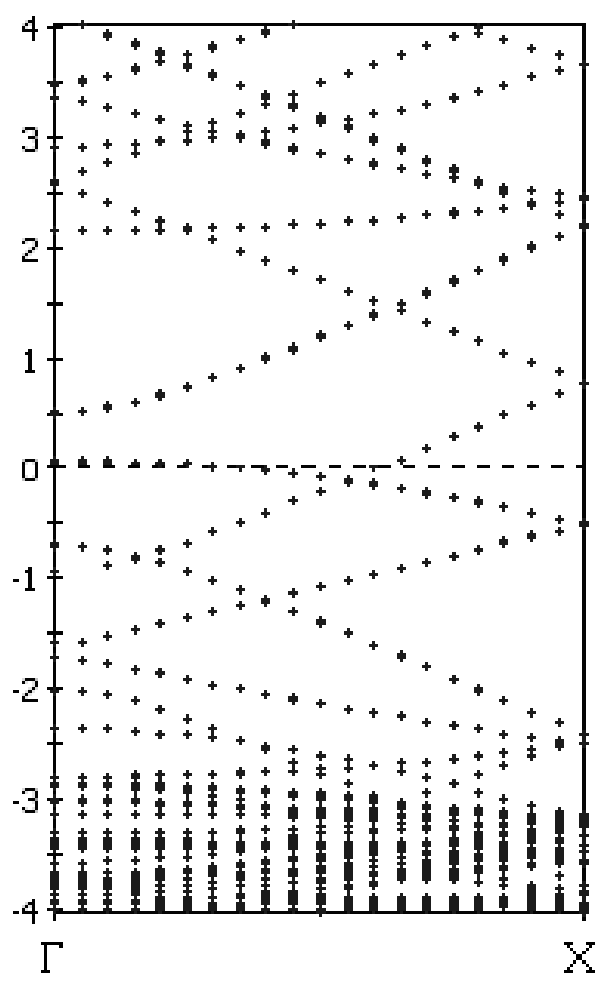




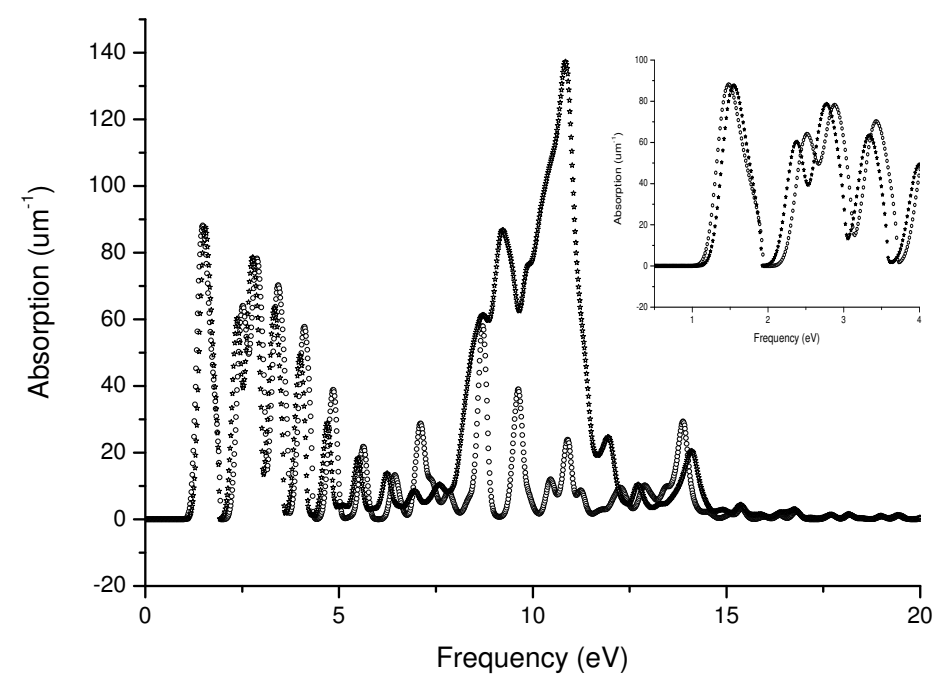

